\title{
Estimação da digestibilidade dos carboidratos não-fibrosos em bovinos utilizando-se o conceito de entidade nutricional em condições brasileiras
}

\section{Edenio Detmann', Sebastião de Campos Valadares Filho ${ }^{1}$, Lara Toledo Henriques², Douglas dos Santos Pina ${ }^{3}$, Mário Fonseca Paulino ${ }^{1}$, Rilene Ferreira Diniz Valadares ${ }^{4}$, Mário Luiz Chizzotti $^{3}$, Karla Alves Magalhães ${ }^{3}$}

\author{
${ }^{1}$ Departamento de Zootecnia, Universidade Federal de Viçosa, Viçosa-MG, 36571-000. Bolsista do CNPq. \\ 2 Pós-Doutoranda, DZO-UFV. Bolsista do CNPq. \\ ${ }^{3}$ Doutorando em Zootecnia - UFV. \\ ${ }^{4}$ Departamento de Veterinária - UFV - Bolsista do CNPq.
}

RESUMO - Objetivou-se neste estudo estimar a fração aparentemente digestível dos carboidratos não-fibrosos (CNF) a partir dos teores dietéticos em bovinos com base no conceito de entidade nutricional. O banco de dados utilizado foi constituído de 167 observações experimentais (57 obtidas de dois experimentos com vacas em lactação e 110 de três experimentos com bovinos em crescimento e terminação). O banco de dados foi aleatoriamente dividido em dois subgrupos, o primeiro utilizado para estimação de parâmetros e o segundo, para validação de equações. Ajustaram-se equações de regressão pelo teste de identidade de modelos por meio de variável indicadora para diferenciação entre categorias animais. O coeficiente de digestibilidade verdadeiro dos CNF $(0,9507)$ não diferiu entre as categorias animais. Verificou-se superioridade da fração metabólica fecal para vacas em lactação. Essa porção mostrou-se não relacionada ao teor dietético de CNF (CNF\%) quando expressa como percentual da dieta. Assim, para animais em condições de alimentação ad libitum, o teor de CNF aparentemente digestíveis $\left(\mathrm{CNF}_{\mathrm{ad}} \%\right)$ pode ser estimado pelas equações: $\mathrm{CNF}_{\mathrm{ad}} \%=0,9507 \mathrm{CNF} \%$ - 5,72 para vacas em lactação; e $\mathrm{CNF}_{\mathrm{ad}} \%=0,9507 \mathrm{CNF} \%-5,11$ para bovinos em crescimento e terminação. O processo de validação indicou similaridade entre valores preditos e observados de $\mathrm{CNF}_{\mathrm{ad}} \%$. O teste de entidade nutricional fornece estimativas não-viesadas para $\mathrm{CNF}$ ad $\%$.

Palavras-chave: equações somativas, digestibilidade aparente, NDT, sistema NRC

\section{Estimation of nonfiber carbohydrates digestibility in cattle using the Lucas test approach under Brazilian conditions}

\begin{abstract}
The objective of this study was to estimate the dietary apparent digestible fraction of nonfiber carbohydrates (NFC) using the Lucas test concept. The dataset contained a total of 167 observations with 57 originated from two trials conducted with lactating cows and 110 from three experiments with growing and finishing cattle. The dataset was randomly divided in two subsets. The first subset was used to estimate the model parameters while the second for model validation. Tests for checking equality of models were conducted using a Dummy variable for comparing animal categories. The true digestibility coefficient did not differ between animal categories, and the estimated value was 0.9705 It was observed greater metabolic fecal NFC for lactating cows than for growing and finishing cattle. The metabolic fecal NFC expressed as a percentile of dietary NFC levels (NFC\%) did not change in this study. Thus, the dietary apparent digestible $\mathrm{NFC}\left(\mathrm{NFC}_{\mathrm{ad}} \%\right)$ can be estimated by the following equations: $\mathrm{NFC}_{\mathrm{ad}} \%=0.9507 \mathrm{NFC} \%-5.72$ for lactating cows and $\mathrm{NFC}_{\mathrm{ad}} \%=0.9507 \mathrm{NFC} \%-5.11$ for growing and finishing cattle. The model validation procedure indicated that observed and estimated values of $\mathrm{NFC}_{\mathrm{ad}} \%$ were not different. It can be concluded that the Lucas test yields reliable estimates of $\mathrm{NFC}_{\mathrm{ad}} \%$.
\end{abstract}

Key Words: apparent digestibility, NRC system, summative equations, total digestible nutrients

\section{Introdução}

A obtenção de estimativas de digestibilidade dos alimentos constitui aspecto básico para o conhecimento de seu valor energético, notadamente via nutrientes digestíveis totais (NDT), permitindo o balanceamento adequado de dietas que propiciem o atendimento das demandas para mantença e produção dos animais.

No entanto, mesmo constituindo parâmetro digestivo estático, ou seja, podendo ser representado por uma estimativa pontual, o acesso à digestibilidade de um alimento ou de seus respectivos componentes químicos constitui 
processo oneroso e demorado, sobretudo quando realizado pelos métodos clássicos in vivo.

Embora métodos alternativos para estimação de parâmetros da digestibilidade tenham sido desenvolvidos em ambientes in situ e in vitro, tanto por vias gravimétricas (Tilley \& Terry, 1963; Goering \& Van Soest, 1975) quanto metabólicas (Schofield et al., 1994), as estimativas têm apresentado vícios substanciais em relação aos valores observados in vivo para alimentos produzidos em condições tropicais (Vieira et al., 2000; Detmann et al., 2005).

De forma alternativa aos métodos biológicos, a utilização da composição química dos alimentos como método de predição da digestibilidade em ruminantes tem sido sugerida por diversos autores (Conrad et al., 1984; Weiss et al., 1992; Van Soest, 1994). Nessa perspectiva, o NRC (2001) propôs um método com esse embasamento para estimação do teor de NDT dos alimentos, o qual se constitui de um sistema de equações somativas, de modo que, a cada grupo de compostos químicos, se destina uma equação responsável pela obtenção de estimativas das frações verdadeiramente digestíveis, com posteriores correções no tocante às perdas metabólicas e ao nível de consumo.

Embora este método seja teoricamente embasado (Conrad et al., 1984; Weiss et al., 1992), não tem apresentado eficiência de predição satisfatória quando aplicado a alimentos obtidos em condições tropicais (Rocha Jr. et al., 2003; Campos, 2004; Detmann et al., 2004; Oliveira, 2005), pois fornece estimativas substancialmente desviadas dos valores observados in vivo.

De forma mais específica, por se tratar de sistema somativo (Weiss et al., 1992; NRC, 2001), as divergências das estimativas de NDT constituem somatório simples das divergências observadas para cada grupo de compostos separadamente (EE, FDN, CNF e PB). Assim, possíveis correções ou alterações sobre o sistema somativo adotado pelo NRC (2001) devem ser investigadas quanto às estimativas individuais de cada grupo de compostos, as quais refletirão diretamente sobre as estimativas obtidas para o nível de NDT do alimento ou da dieta.

Como recurso para predição energética a partir da composição química dietética, os fundamentos do teste de entidade nutricional ou teste de Lucas (Lucas \& Smart, 1959) têm sido utilizados para obtenção de estimativas do teor de CNF digestíveis de alimentos ou dietas (Van Soest, 1994; NRC, 2001). Neste contexto, segundo proposições de Lucas \& Smart (1959), componentes dos alimentos com comportamento digestivo homogêneo, como os CNF, poderiam ter suas estimativas de digestibilidade aparente obtidas a partir de seu coeficiente de digestibilidade verdadeiro e de sua contribuição metabólica fecal.
Mesmo que esses fundamentos estejam incorporados ao sistema somativo adotado pelo NRC (2001), o qual possui relativa acurácia para alimentos produzidos em regiões temperadas (Weiss et al., 1992), as estimativas do teor de CNF digestíveis obtidas em condições tropicais tem se mostrado significativamente desviadas do valores observados in vivo (Rocha Jr. et al., 2003; Campos, 2004; Oliveira, 2005; Pina, 2005), justificando a re-avaliação desse método para predição da contribuição dos CNF ao NDT em alimentos utilizados em condições tropicais.

Dessa forma, objetivou-se neste estudo avaliar o teste de entidade nutricional como estimador do teor de CNF digestíveis, em animais alimentados em condições tropicais.

\section{Material e Métodos}

As avaliações foram realizadas utilizando-se um banco de dados resultante de dois experimentos realizados com vacas em lactação (Chizotti, 2004; Pina, 2005) e três com bovinos em crescimento e terminação (Rennó, 2003; Magalhães, 2003; Chizotti, 2004), totalizando 57 e 110 observações, respectivamente, para cada categoria animal.

Foram levantadas informações relativas ao teor dietético ( $\%$ da MS), ao consumo ( $\mathrm{kg} /$ dia $)$ e à digestibilidade aparente total (\%) dos CNF (Tabela 1). Em todos os experimentos avaliados, os animais foram alimentados ad libitum. Os teores de CNF em amostras de alimentos, sobras e fezes foram avaliados por meio da equação:

$$
C N F=100-(P B+E E+M M+F D N c p)
$$

em que: $\mathrm{CNF}=$ teor estimado de $\mathrm{CNF}(\%) ; \mathrm{PB}=$ teor de $\mathrm{PB}$ $(\%) ; \mathrm{EE}=$ teor de $\mathrm{EE}(\%) ; \mathrm{MM}=$ teor de $\mathrm{MM}$; e FDNcp = teor de FDN corrigido para cinzas e proteína (\%). Em todas as análises para avaliação dos teores de FDN, utilizou-se amilase termoestável para solubilização de compostos amiláceos (Mertens, 2002).

No caso das dietas nas quais se utilizou uréia como fonte de compostos nitrogenados não-protéicos, os teores dietéticos de CNF foram estimados por adaptação à proposição de Hall (2000):

$C N F=100-[(P B-P B u+U)+E E+M M+F D N c p]$

em que: $\mathrm{PBu}=$ teor de $\mathrm{PB}$ proveniente da uréia (\%); e $\mathrm{U}=$ teor de uréia $(\%)$.

Em termos analíticos, o conjunto total de dados foi aleatoriamente fracionado em dois subgrupos: o primeiro destinado à estimação de parâmetros e o segundo à validação do modelo (Tabela 1) (Mertens, 1976). A casualização do processo de divisão do conjunto de dados foi conduzida de forma restrita, de acordo com as categorias animais. 
Tabela 1 - Estatísticas descritivas para o teor de CNF na dieta (CNF - \% da MS), o consumo diário (CCNF - kg) e a digestibilidade aparente total dos CNF (DCNF - \%)

Table 1 - Descriptive statistics of NFC level in diet (NFC - \% of DM), NFC daily intake (NFCl - kg), and total apparent digestibility of NFC (NFCD - \%)

\begin{tabular}{|c|c|c|c|c|c|c|}
\hline \multirow{3}{*}{$\begin{array}{l}\text { Estatística } \\
\text { Statistic } \\
\end{array}$} & \multicolumn{3}{|c|}{$\begin{array}{l}\text { Vacas em lactação } \\
\text { Lactating cows }\end{array}$} & \multicolumn{3}{|c|}{$\begin{array}{l}\text { Bovinos em crescimento e terminação } \\
\text { Growing and finishing cattle }\end{array}$} \\
\hline & $\mathrm{CNF}$ & $\mathrm{CCNF}$ & DCNF & CNF & CCNF & DCNF \\
\hline & $N F C$ & NFCI & $N F C D$ & $N F C$ & $N F C$ & $I N F C D$ \\
\hline \multicolumn{7}{|c|}{$\begin{array}{c}\text { Conjunto total de dados } \\
\text { Complete dataset }\end{array}$} \\
\hline Média (Mean) & 39,10 & 7,078 & 80,30 & 40,01 & 2,814 & 82,65 \\
\hline Máximo (Maximum) & 49,11 & 9,270 & 89,98 & 49,49 & 5,164 & 92,41 \\
\hline Mínimo (Minimum) & 31,18 & 3,208 & 59,51 & 19,83 & 0,480 & 67,16 \\
\hline Desvio-padrão (Standard deviation) & 5,56 & 1,496 & 7,31 & 3,98 & 0,886 & 5,37 \\
\hline $\mathrm{n}$ & 57 & & 110 & & & \\
\hline \multicolumn{7}{|c|}{$\begin{array}{l}\text { Dados para estimação de parâmetros } \\
\text { Data for parameters estimation }\end{array}$} \\
\hline Média (Mean) & 39,56 & 7,221 & 80,29 & 40,51 & 3,218 & 82,34 \\
\hline Máximo (Maximum) & 49,00 & 9,270 & 89,98 & 46,29 & 5,164 & 89,87 \\
\hline Mínimo (Minimum) & 31,18 & 3,272 & 61,26 & 33,74 & 1,471 & 67,16 \\
\hline Desvio-padrão (Standard deviation) & 5,37 & 1,614 & 7,79 & 2,71 & 0,659 & 5,25 \\
\hline $\mathrm{n}$ & 29 & & 55 & & & \\
\hline \multicolumn{7}{|c|}{$\begin{array}{l}\text { Dados para validação } \\
\text { Data for model validation }\end{array}$} \\
\hline Média (Mean) & 38,62 & 6,930 & 80,32 & 39,52 & 2,411 & 82,95 \\
\hline Máximo (Maximum) & 49,11 & 9,268 & 89,24 & 49,49 & 3,731 & 92,41 \\
\hline Mínimo (Minimum) & 31,92 & 3,208 & 59,51 & 19,83 & 0,480 & 67,37 \\
\hline Desvio-padrão (Standard deviation) & 5,80 & 1,377 & 6,93 & 4,92 & 0,906 & 5,51 \\
\hline $\mathrm{n}$ & 28 & & 55 & & & \\
\hline
\end{tabular}

O procedimento de construção do bancos de dados utilizado neste trabalho (estimação e validação) pode ser considerado menos rigoroso em processos de validação de modelos (Mertens, 1976) em relação a outros procedimentos comumente adotados (Detmann et al., 2006). Contudo, sua adoção justifica-se pela carência de banco de dados nos quais se encontram disponíveis estimativas de consumo e digestibilidade de CNF obtidas a partir de teores de FDNcp (Equações 1 e 2) e com correções para a presença de uréia nas rações (Equação 2)

Anteriormente à utilização dos dados, procedeu-se à avaliação da homogeneidade das variâncias residuais entre experimentos por meio do teste de Bartlett (Snedecor \& Cochran, 1989), verificando-se condição de homocedasticidade $(\mathrm{P}>0,05)$.

Os dados referentes ao subconjunto destinado à estimação de parâmetros foram adaptados ao teste de entidade nutricional proposto por Lucas \& Smart (1959), que se baseia no ajustamento do seguinte modelo de regressão linear simples:

$Y_{i}=\beta X_{i}+\alpha+\varepsilon_{i}$

em que: $\mathrm{Y}_{\mathrm{i}}=\mathrm{CNF}$ aparentemente retidos ou digeridos $(\mathrm{kg} /$ dia); $X_{i}=$ CNF ingeridos $(\mathrm{kg} /$ dia $) ; \beta=$ coeficiente de digestibilidade verdadeiro dos CNF; $\alpha=$ CNF metabólicos fecais $(\mathrm{kg} / \mathrm{dia})$; e $\varepsilon_{\mathrm{i}}=$ erro aleatório não-observável, pressuposto NID $\left(0 ; \sigma^{2}\right)$.

Para que o componente químico do alimento/dieta possa ser considerado entidade nutricional, validando-se, portanto, o processo de estimação, as seguintes restrições devem ser observadas (Lucas \& Smart, 1959):

$0 \leq \hat{\beta} \leq 1$

$\hat{\alpha} \leq 0$

Em virtude da possível diferença entre as categorias animais avaliadas, aplicou-se à equação (3) parametrização com variáveis indicadoras (Draper \& Smith, 1966) para se testar a identidade de modelos em relação às categorias animais. Por outro lado, inseriu-se no modelo um componente aleatório relativo à variação inter-experimentos. Desta forma, redefiniu-se o modelo descrito em (3) como:

$Y_{i j k}=\beta_{1} X_{i j k}+\beta_{2} C X_{i j k}+\alpha_{1}+\alpha_{2} C+E_{(i) k}+\varepsilon_{i j k}$

em que: $\mathrm{Y}_{\mathrm{ijk}}=\mathrm{CNF}$ aparentemente retidos obtidos na observação $\mathrm{j}$, obtida no experimento $\mathrm{k}$ com a categoria animal i $(\mathrm{kg} / \mathrm{dia}) ; \mathrm{X}_{\mathrm{ijk}}=\mathrm{CNF}$ consumidos, obtidos na observação $\mathrm{j}$, no experimento $\mathrm{k}$ com a categoria animal i; $\mathrm{C}=$ variável indicadora da categoria animal vacas em lactação, 
sendo $C=0$ para animais em crescimento e terminação e $C=1$ para vacas em lactação; $\beta_{1}$ e $\beta_{2}=$ parâmetros que definem o coeficiente de digestibilidade verdadeiro dos CNF; $\alpha_{1}$ e $\alpha_{2}=$ parâmetros que definem os CNF metabólicos fecais $(\mathrm{kg} / \mathrm{dia}) ; \mathrm{E}_{\mathrm{i}) \mathrm{k}}=$ efeito aleatório relativo ao experimento $\mathrm{k}$ aninhado à categoria animal $\mathrm{i}$; e $\varepsilon_{\mathrm{ijk}}=$ erro aleatório não-observável, pressuposto $\operatorname{NID}\left(0 ; \sigma^{2}\right)$.

As observações relativas aos $\mathrm{CNF}$ aparentemente retidos foram obtidas pelo produto entre o consumo e o coeficiente de digestibilidade aparente.

A partir das modificações propostas para o modelo (3), descritas em (6), definiram-se as restrições para a consideração de entidade nutricional:

$0 \leq \hat{\beta_{1}}+\hat{\beta_{2}} \cdot C \leq 1$

$\hat{\alpha}_{1}+\hat{\alpha}_{2} \cdot C \leq 0$

A partir dos níveis dietéticos de CNF verificados no subconjunto de dados destinado à validação do modelo, foram estimados os teores dietéticos de CNF aparentemente digestíveis por meio das estimativas do coeficiente de digestibilidade verdadeiro e da contribuição metabólica fecal dos CNF.

$\mathrm{Na}$ análise estatística, procedeu-se ao ajustamento do modelo de regressão linear simples de valores observados (variável dependente) sobre valores preditos (variável independente), conduzindo-se os testes estatísticos sob as seguintes hipóteses (Mayers et al., 1994):

$$
H_{0}: \beta_{0}=0 \text { e } \beta_{1}=1 \times H_{a}: n \tilde{a} o H_{0}
$$

Para o caso de não-rejeição da hipótese de nulidade, conclui-se pela similaridade entre valores preditos e observados. Todos os procedimentos estatísticos foram realizados por meio do programa SAS (Statistical Analysis System), adotando-se 0,05 como nível crítico de probabilidade para o erro tipo I.

\section{Resultados e Discussão}

As bases adotadas para o desenvolvimento do modelo foram adaptadas às proposições de Blaxter \& Mitchell (1948) e Lucas (1960), as quais se referem à dinâmica digestiva dos compostos nitrogenados. Neste contexto, após a ingestão e digestão, pode-se resumir a massa aparentemente não-digerida de $\mathrm{CNF}$ como:

$C N F f=C N F i+C N F m+C N F e$

em que: $\mathrm{CNFf}=\mathrm{CNF}$ fecais $(\mathrm{kg} / \mathrm{dia}) ; \mathrm{CNFi}=\mathrm{CNF}$ verdadei ramente indigeridos $(\mathrm{kg} / \mathrm{dia}) ; \mathrm{CNFm}=\mathrm{CNF}$ metabólicos $(\mathrm{kg} / \mathrm{dia}) ; \mathrm{e} \mathrm{CNFe}=\mathrm{CNF}$ endógenos $(\mathrm{kg} / \mathrm{dia})$.

Definiu-se como CNF metabólicos a porção oriunda de secreções do trato digestivo (Lucas, 1960), acrescida de matéria microbiana (Van Soest, 1994), e, como CNF endógenos, a fração fecal advinda da excreção de "restos" metabólicos pelas células do trato gastrintestinal (Lucas, 1960).

A partir destas definições, a identidade exposta em (9) foi relacionada ao consumo diário de CNF:

$$
\begin{aligned}
& C N F c-C N F f=C N F c-(C N F i+C N F m+C N F e) \\
& C N F a r=C N F c-C N F i-C N F m-C N F e \\
& \frac{C N F a r}{C N F c}=\frac{C N F c-C N F i-C N F m-C N F e}{C N F c} \\
& D a_{C N F}=1-\frac{C N F i}{C N F c}-\frac{C N F m}{C N F c}-\frac{C N F e}{C N F c}
\end{aligned}
$$

em que: $\mathrm{CNFc}=\mathrm{CNF}$ consumidos $(\mathrm{kg} / \mathrm{dia}) ; \mathrm{CNFar}=\mathrm{CNF}$ aparentemente retidos ou digeridos $(\mathrm{kg} / \mathrm{dia}) ; \mathrm{Da}_{\mathrm{CNF}}=$ coeficiente de digestibilidade aparente dos CNF; e CNFf, $\mathrm{CNFi}, \mathrm{CNFm}, \mathrm{CNFe}=$ como definidos anteriormente.

Em adaptação às proposições de Blaxter \& Mitchell (1948), pode-se afirmar que a contribuição endógena dos CNF pode ser representada como uma função matemática que expressa proporcionalidade à massa metabólica do animal:

$\frac{C N F e}{C N F c}=\frac{\varepsilon \cdot P^{\frac{3}{4}}}{C N F c}=\frac{\varepsilon}{C N F c} \cdot P^{\frac{3}{4}}$

em que: $\mathrm{P}=$ massa do animal $(\mathrm{kg}) ; \mathrm{e}=$ constante relacionada à liberação endógena de $\mathrm{CNF}$ no trato gastrintestinal por unidade de massa metabólica $\left(\mathrm{kg} / \mathrm{kg} \times \mathrm{dia}^{-1}\right)$; e CNFe e $\mathrm{CNFc}=$ como definidos anteriormente.

A razão $/ \mathrm{CNFc}$, expressa em (11), poderia ser considerada significativa somente se o consumo de CNF assumisse valores extremamente pequenos (Lucas, 1960), possivelmente em níveis de alimentação abaixo da mantença. Assim, em condições "normais" de alimentação (mantença ou produção), ter-se-ia:

$$
\lim _{C N F C \rightarrow C N F^{\circ} c} \frac{\varepsilon}{C N F C} \cdot P^{\frac{3}{4}}=0
$$

em que: $\mathrm{CNF}^{\circ} \mathrm{c}=$ consumo de $\mathrm{CNF}$ em condições "normais" de alimentação $(\mathrm{kg} / \mathrm{dia})$; e CNFc, $\varepsilon$ e $\mathrm{P}=$ como definidos anteriormente.

Dessa forma, rescreveu-se a equação (10d) como:

$$
\begin{aligned}
& D a_{C N F}=\left(1-\frac{C N F i}{C N F c}\right)-\frac{C N F m}{C N F c} \\
& D a_{C N F}=\left(1-I v_{C N F}\right)-\frac{C N F m}{C N F c} \\
& D a_{C N F}=D v_{C N F}-\frac{C N F m}{C N F c}
\end{aligned}
$$

em que: $I v_{\mathrm{PB}}=$ coeficiente de indigestibilidade verdadeiro dos $\mathrm{CNF}$; $\mathrm{D} v_{\mathrm{CNF}}=$ coeficiente de digestibilidade verdadeiro dos $\mathrm{CNF}$; e $\mathrm{Da}_{\mathrm{CNF}}, \mathrm{CNFi}, \mathrm{CNF}$ e $\mathrm{CNFm}=$ como definidos anteriormente. 
Multiplicando-se ambos os termos da equação (13c) pelo consumo de CNF, obteve-se:

$$
C N F c \cdot D a_{C N F}=C N F c \cdot D v_{C N F}-C N F m
$$

Derivando-se (14) em função do consumo de CNF, encontra-se:

$$
\frac{d\left(\mathbf{C N F c} \cdot D a_{C N F}\right)}{d C N F c}=D v_{C N F}-\frac{d C N F m}{d C N F c}
$$

Dessa forma, a diferencial expressa em (15), que corresponde ao coeficiente de digestibilidade aparente dos $\mathrm{CNF}$, pode ser composta por dois diferentes componentes: o primeiro representado pelo coeficiente de digestibilidade verdadeiro constante dos CNF e o segundo, por uma porção metabólica que se diferencia pela massa de CNF ingerida.

Convertendo-se a equação (15) para a base percentual dietética, encontrou-se

$$
\begin{aligned}
& C N F(\%) \cdot D a_{C N F}=C N F(\%) \cdot D v_{C N F}-C M_{C N F} \\
& C N F_{a d}(\%)=C N F_{v d}(\%)-C M_{C N F}
\end{aligned}
$$

em que: $\mathrm{CNF}(\%)=$ nível dietético de $\mathrm{CNF} ; \mathrm{CM}_{\mathrm{CNF}}=$ contribuição metabólica de CNF como percentual da MS dietética; $\mathrm{CNF}_{\mathrm{ad}}(\%)=$ nível dietético de $\mathrm{CNF}$ aparentemente digestíveis, como percentual da MS; e $\mathrm{CNF}_{\mathrm{vd}}(\%)=$ nível dietético de CNF verdadeiramente digestíveis, como percentual da MS.

Com base na significância observada para a variável indicadora incluída na equação (6), pode-se inferir que não houve efeitos de categoria animal sobre o coeficiente de inclinação da relação entre CNF ingeridos e aparentemente retidos $(\mathrm{P}>0,16)$. Este resultado indica que a digestibilidade verdadeira deste componente, representada pelo coeficiente de inclinação expresso em (6), não depende da categoria animal. Desta forma, segundo as estimativas obtidas, verificou-se a equação:

$$
C N F_{v d}(\%)=0,9507 \cdot C N F(\%)
$$

em que: 0,9507 = estimativa para o coeficiente de digestibilidade verdadeiro dos $\mathrm{CNF}$, com $\operatorname{IC}(\beta)_{95 \%}$ : $[0,8742 \leq \beta \leq 1,0272]$; sendo os demais termos definidos anteriormente.

A acurácia dessa estimativa pôde ser confirmada pela alta agregação dos pontos referentes às informações do banco de dados construído neste estudo para ambas as categorias animais avaliadas $\left(\mathrm{R}^{2}=0,9865\right)$, mesmo com a adoção de um coeficiente de inclinação conjunto (Figuras 1 e 2).

A projeção dos limites do intervalo de confiança a 95\% de probabilidade para o coeficiente de digestibilidade verdadeiro dos CNF assemelhou-se à variação das estimativas deste parâmetro $(0,85$ a 1,20$)$ obtidas pelo teste de entidade nutricional, relatadas por Van Soest (1994). Verificou-se,

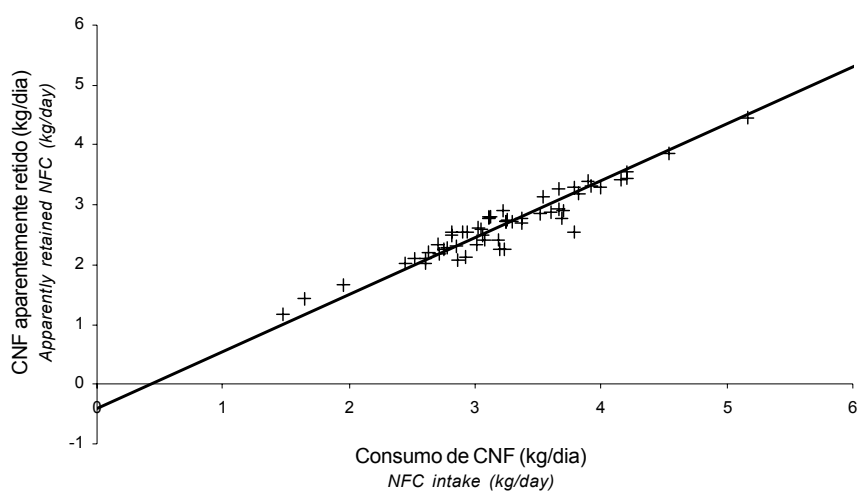

Figura 1 - Relação entre consumo e quantidade aparentemente retida de CNF em bovinos em crescimento e terminação $\left(\hat{Y}=-0,410+0,9507 X ; R^{2}=0,9865\right)$.

Figure 1 - Relationship between intake and apparently retained NFC in growing and finishing cattle (the tendency line corresponds to the least squares straight line).

porém, que o valor paramétrico 0,98 , assumido como coeficiente de digestibilidade verdadeiro pelo sistema somativo proposto por Weiss et al. (1992), encontra-se contido no referido intervalo de confiança, indicando similaridade entre a estimativa obtida neste trabalho e aquela adotada pelo sistema NRC (2001) ( $\mathrm{P}>0,05)$.

Em contrapartida, observou-se efeito significativo $(\mathrm{P}<0,01)$ de categoria animal sobre a massa de CNF metabólicos fecais, cujas estimativas obtidas diretamente pelo modelo descrito em (6) foram de 0,410 e 0,991 kg/dia, para bovinos em crescimento e terminação e vacas em lactação, respectivamente (Figuras 1 e 2). Ressalta-se que as estimativas obtidas para o coeficiente de digestibilidade verdadeiro e as contribuições metabólicas enquadraram-se nas restrições impostas em (7) e (8), garantindo o status de entidade nutricional aos CNF (Lucas \& Smart, 1959; Van Soest, 1994).

A superioridade da fração metabólica dos CNF em vacas em lactação, em comparação a animais em crescimento e terminação $(\mathrm{P}<0,01)$ (Figuras $1 \mathrm{e} 2$ ), pode ser suportada por sua relação diretamente proporcional ao consumo de CNF (Lucas, 1960) (Tabela 1).

A verificação de um coeficiente de digestibilidade verdadeiro constante e independente da categoria animal (Equação 17), associado à contribuição metabólica diferenciada em função do consumo de CNF (reflexo direto da diferença entre categorias animais; Tabela 1 e Figuras 1 e 2) converge às pressuposições teóricas sumariadas na equação (15), garantindo a validade do modelo proposto neste trabalho.

Com vistas à estimação do teor de CNF aparentemente digestível, que constitui componente direto do teor de NDT da dieta, procedeu-se à conversão das estimativas de CNF 


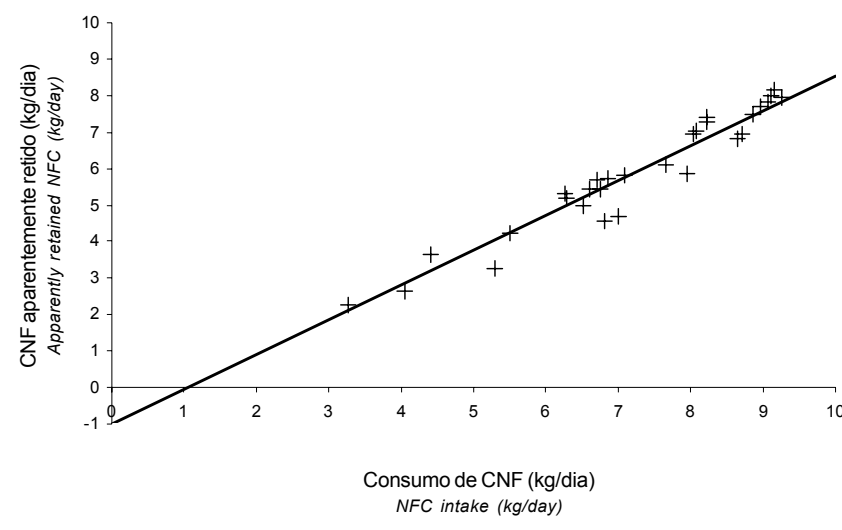

Figura 2 - Relação entre consumo e quantidade aparentemente retida de carboidratos não-fibrosos em vacas em lactação ( $\left.\hat{Y}=-0,991+0,9507 X ; R^{2}=0,9865\right)$.

Figure 2 - Relationship between intake and apparently retained nonfiber carbohydrates in lactating cows (the tendency line corresponds to least squares straight line).

metabólicos ( $\mathrm{kg} / \mathrm{dia})$ em função do teor dietético para cada valor integrante do conjunto de dados utilizado para estimação de parâmetros utilizando-se a equação:

$C N F_{m}(\%)=\frac{C N F_{v d}-C N F a r}{C N F c} \cdot C N F(\%)$

(18a);

$C N F_{m}(\%)=\frac{0,9507 \cdot C N F c-D a_{C N F} \cdot C N F c}{C N F c} \cdot C N F(\%)$

$(18 b)$;

$C N F_{m}(\%)=\left(0,9507-D a_{C N F}\right) \cdot C N F(\%)$

em que: $\mathrm{CNF}_{\mathrm{m}}(\%)=\mathrm{CNF}$ metabólicos, como percentual da MS dietética; sendo os demais termos definidos anteriormente.

A avaliação dos resultados obtidos via equação (18c) para cada categoria animal indicou não haver relação contribuição metabólica percentual x nível dietético de CNF $(\mathrm{P}>0,24)$, o que permite a representação dessa fração por valores constantes, conforme a seguir:

$C N F_{a d}(\%)=0,9507 \cdot C N F(\%)-5,11$ (bovinos em crescimento e terminação)

$C N F_{a d}(\%)=0,9507 \cdot C N F(\%)-5,72($ vacas em lactação)

(19b);

em que: 5,11 e 5,72 = estimativas para CNF metabólicos, como percentual da MS da dieta; sendo os demais termos definidos anteriormente.

Considerando as características do banco de dados utilizado neste estudo, as estimativas obtidas pelas equações (19a) e (19b) são aplicáveis a animais submetidos a consumo ad libitum, ou seja, em nível de produção. Segundo observações realizadas em condições brasileiras, a relação entre o nível de nutrientes digestíveis totais em situações de produção e mantença (consumo restrito) aproxima-se de 0,95 (Costa et al., 2005).
Segundo as relações obtidas neste trabalho (Figuras 1 e 2), não se esperam alterações no coeficiente de digestibilidade verdadeiro dos $\mathrm{CNF}$ em função do nível de consumo, diferenciação observada somente sobre a contribuição metabólica fecal (Equação 15). Portanto, considerando o consumo médio de CNF observado para cada categoria, no tocante ao conjunto de dados para estimação de parâmetros (Tabela 1), e a relação de digestibilidade nos níveis de produção e mantença de 0,95 , obtiveram-se as estimativas do teor dietético de CNF aparentemente digeridos em condições de mantença $\left(\mathrm{CNF}_{\mathrm{adm}}\right)$ :

$C N F_{\text {adm }}(\%)=0,9507 \cdot C N F(\%)-3,35$ (bovinos em crescimento e terminação)

$C N F_{\text {adm }}(\%)=0,9507 \cdot C N F(\%)-4,05($ vacas em lactação $)$

Apesar da concordância entre o coeficiente de digestibilidade verdadeiro dos CNF adotado pelo NRC (2001) e a estimativa obtida neste trabalho (Equação 17), verificou-se discrepância entre as estimativas de contribuição metabólica em nível de mantença obtidas neste estudo (Equações 19a e 19b) e aquela relatada por Weiss et al. (1992) (3,1\%), o que pode constituir, ao menos em parte, causa da divergência entre valores preditos e observados do teor de CNF aparentemente digestíveis em condições tropicais (Rocha Jr. et al., 2003; Campos, 2004; Oliveira, 2005; Pina, 2005).

A discrepância entre estimativas de contribuição metabólica dos CNF pode ser atribuída, em grande parte, a divergências no nível de consumo dos animais (Equação 15 ), as quais podem ser diretamente mensuradas pela diferença no consumo entre categorias animais (Equações 19a e 19b), que não é contemplada pelo modelo proposto por Weiss et al. (1992) e adotado pelo NRC (2001).

Por outro lado, a dimensão da fração metabólica fecal é diretamente influenciada pelo fluxo de nutrientes ao intestino grosso terminal, o qual implica alterações na atividade microbiana cecal (Ørskov, 1988), e pelo nível de componentes fibrosos na dieta (Arroyo-Aguilu \& Evans, 1972; citados por Weiss et al., 1992), condições notadamente divergentes entre animais alimentados em condições tropicais e temperadas.

As estimativas dos parâmetros de regressão, associadas aos respectivos níveis descritivos de probabilidade para os testes de hipóteses, são apresentadas na Tabela 2.

Verificou-se a não-rejeição da hipótese de nulidade avaliada ( $\mathrm{P}>0,11)$ (Tabela 2; Figura 3), sugerindo a adequação do conceito de entidade nutricional e das equações estabelecidas para as categorias animais avaliadas (18a e 18b).

Embora a inspeção gráfica do comportamento dos dados (Figura 3) permita evidenciar certo nível de variação 
Tabela 2 - Equação de validação ajustada $\left(\hat{Y}_{i}=\hat{\beta}_{0}+\hat{\beta}_{1} X_{i}\right)$, valor-P para o teste da hipótese $\mathrm{H}_{0}: \beta_{0}=0$ e $\beta_{1}=1$, desvio-padrão residual $\left(\mathrm{s}_{\mathrm{xy}}\right)$ e número total de pares de valores $(n)$ para a relação entre valores preditos e observados para os níveis dietéticos de CNF aparentemente digestíveis

Table 2 - $\quad$ Adjusted validation equation $\left(\hat{Y}_{i}=\hat{\beta}_{0}+\hat{\beta}_{1} X_{i}\right)$, P-value for testing the hypothesis $H_{0}$ : $\beta_{0}=0$ e $\beta_{1}=1$, residual standard deviation $\left(s_{x y}\right)$ e total number of paired values $(n)$ for the relationship between predicted and observed values of dietary levels of apparently digestible NFC

\begin{tabular}{lc}
\hline Equação ajustada & $\hat{\mathrm{Y}}=-0,7442+1,0368 \mathrm{X}_{\mathrm{i}}$ \\
$\begin{array}{l}\text { Adjusted equation } \\
\text { Valor-P }\end{array}$ & \\
$P$-value & 0,1139 \\
$\mathrm{~S}_{\mathrm{xy}}$ & 2,016 \\
$\mathrm{n}$ & 83 \\
\hline
\end{tabular}

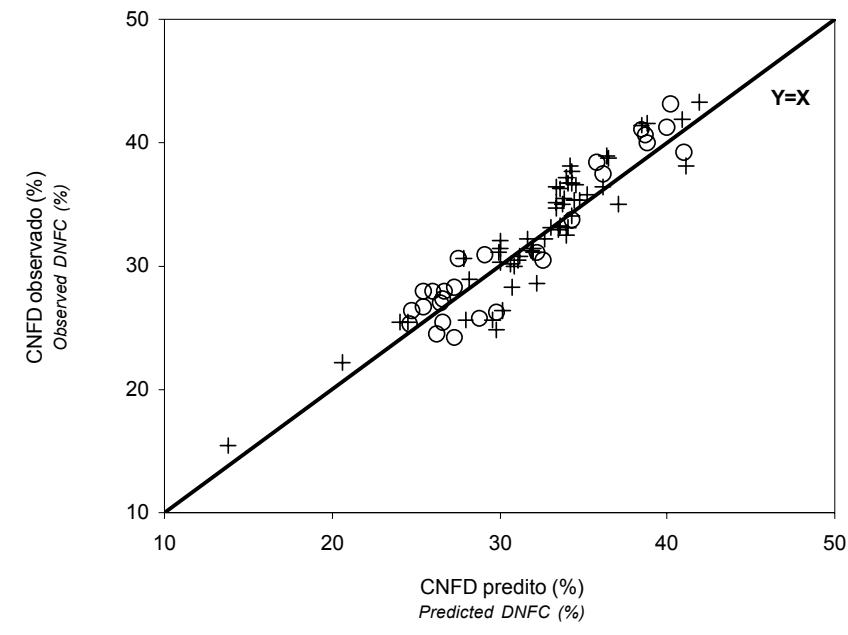

Figura 3 - Relação entre valores preditos e observados para os teores de carboidratos não-fibrosos digestíveis (CNFD) na dieta $(+=$ bovinos em crescimento e terminação; o = vacas em lactação).

Figure 3 - Relationship between predicted and observed values for digestible nonfiber carbohydrates (DNFC) $++=$ growing and finishing cattle; $\mathrm{o}=$ lactating cows).

dos dados em torno da linha de igualdade ( $\mathrm{Y}=\mathrm{X})$, este nível, no entanto, não causou discrepância significativa entre valores preditos e observados $(\mathrm{P}>0,11)$ (Tabela 2$)$. Entende-se que esse comportamento decorra de variações no consumo total de CNF inerentes a cada animal, o que resulta diretamente em variações na porção metabólica fecal (Equação 15). Como as equações propostas basearam-se em valores médios (18a e 18b), cujo conjunto de dados empregado para estimação não permitiu evidenciar relações ( $\mathrm{P}>0,24)$ com o nível dietético de $\mathrm{CNF}$, essas variações mostram-se pouco relevantes e não-comprometedoras ao processo de estimação.
A acurácia obtida pelo modelo proposto neste trabalho evidenciou a capacidade do teste de entidade nutricional em predizer o teor dietético de CNF digestíveis em alimentos produzidos em condições tropicais, podendo incrementar a exatidão das estimativas dos teores de NDT a partir da composição química dos alimentos ou das dietas.

\section{Conclusões}

As estimativas da fração digestível dos carboidratos não-fibrosos obtidas pela aplicação do conceito de entidade nutricional mostraram-se acuradas para alimentos/dietas produzidos em condições tropicais.

\section{Literatura Citada}

BLAXTER, K.L.; MITCHELL, H.H. The factorization of the protein requeriments of ruminants and of the protein values of feeds, with particular reference to the significance of metabolic fecal nitrogen. Journal of Animal Science, v.7, n.2, p.351-372, 1948.

CAMPOS, P.R.S.S. Estimativas do valor energético de alguns volumosos e da digestibilidade da fibra em detergente neutro em ovinos. Viçosa, MG: Universidade Federal de Viçosa, 2004. 67p. Dissertação (Mestrado em Zootecnia) - Universidade Federal de Viçosa, 2004.

CHIZZOTTI, M.L. Avaliação da casca de algodão para novilhos de origem leiteira e determinação da excreção de cretinina e produção de proteína microbiana em novilhas e vacas. Viçosa, MG: Universidade Federal de Viçosa, 2004. 132p. Dissertação (Mestrado em Zootecnia) - Universidade Federal de Viçosa, 2004.

CONRAD, H.R.; WEISS, W.P.; ODWONGO, W.O. et al. Estimating net energy lactation from components of cell solubles and cell walls. Journal of Dairy Science, v.67, n.2, p.427-436, 1984.

COSTA, M.A.L; VALADARES FILHO, S.C.; VALADARES, R.F.D et al. Validação das equações do NRC (2001) para predição do valor energético de alimentos em condições brasileiras. Revista Brasileira de Zootecnia, v.34, n.1, p.280-287, 2005.

DETMANN, E.; ZERVOUDAKIS, J.T.; CABRAL, L.S. et al. Validação de equações preditivas da fração indigestível da fibra em detergente neutro em gramíneas tropicais. Revista Brasileira de Zootecnia, v.33, n.6, p.1866-1875, 2004 (supl. 1).

DETMANN, E.; PAULINO, M.F.; CABRAL, L.S. et al. Simulação e validação de parâmetros da cinética digestiva em novilhos mestiços suplementados a pasto por intermédio de sistema in vitro de produção de gases. Revista Brasileira de Zootecnia, v.34, n.6, p.3112-2122, 2005.

DETMANN, E.; VALADARES FILHO, S.C.; CAMPOS, J.M.S. et al. Estimação da digestibilidade do extrato etéreo em ruminantes a partir dos teores dietéticos: desenvolvimento de um modelo em condições brasileiras. Revista Brasileira de Zootecnia, v.35, n.4, p.1469-1478, 2006.

DRAPER, N.; SMITH, H. Applied regression analysis. New York: John Willey \& Sons, 1966. 407p.

GOERING,H.K.; Van SOEST, P.J. Forage fiber analyses (apparatus, reagents, procedures, and some applications). Washington: USDA, 1975. 20p. (Agricultural handbook, 379)

HALL, M.B. Neutral detergent-soluble carbohydrates. Nutritional relevance and analysis. Gainesville: University of Florida, 2000. 76p. 
LUCAS, H.L. Relations between apparent digestibility and the composition of feed and feces. 1. A quantitative theory. Raleigh: North Caroline State College, 1960. 55p (Technical Report).

LUCAS, H.L.; SMART, W.W.G. Chemical composition and the digestibility of forages. In: PASTURE AND CROP IMPROVEMENT CONFERENCE, 16., 1959, Mississipi. Proceedings... Mississipi: Mississipi State University, 1959. p.23-26.

MAGALHÃES, K.A. Níveis de uréia ou casca de algodão na alimentação de novilhos de origem leiteira em confinamento. Viçosa, MG: Universidade Federal de Viçosa, 2003. 89p. Dissertação (Mestrado em Zootecnia) - Universidade Federal de Viçosa, 2003.

MAYER, D.G.; STUART, M.A.; SWAIN, A.J. Regression of realworld data on model output: an appropriate overall test of validity. Agricultural Systems, v.45, n.1, p.93-104, 1994.

MERTENS, D.R. Principles of modeling and simulation in teaching and research. Journal of Dairy Science, v.60, n.7, p.11761186, 1976.

MERTENS, D.R. Gravimetric determination of amylase-treated neutral detergent fiber in feeds with refluxing in beakers or crucibles: collaborative study. Journal of AOAC International, v.85, n.6, p.1217-1240, 2002.

NATIONAL RESEARCH COUNCIL - NRC. Nutrient requirements of dairy cattle. 7.ed. Washington, D.C.: Academic Press, 2001. 381p.

OLIVEIRA, A.S. Casca de café ou casca de soja em substituição ao milho em dietas à base de cana-de-açúcar para vacas leiteiras . Viçosa, MG: Universidade Federal de Viçosa, 2005. 90p. Dissertação (Mestrado em Zootecnia) - Universidade Federal de Viçosa, 2005.

ØRSKOV, E.R. Nutrición proteica de los rumiantes. Zaragoza: Acribia, 1988. $178 \mathrm{p}$

PINA, D.S. Fontes de proteína para vacas em lactação. Viçosa, MG: Universidade Federal de Viçosa, 2005. 84p. Dissertação (Mestrado em Zootecnia) - Universidade Federal de Viçosa, 2005.
RENNÓ, L.N. Consumo, digestibilidade total e parcial, produção microbiana, parâmetros ruminais e excreções de uréia e creatinina em novilhos alimentados co dietas contendo quatro níveis de uréia ou dois níveis de proteína. Viçosa, MG: Universidade Federal de Viçosa, 2003. 267p. Tese (Doutorado em Zootecnia) - Universidade Federal de Viçosa, 2003.

ROCHA JR., V.R.; VALADARES FILHO, S.C.; BORGES, A.M. et al Estimativa do valor energético dos alimentos e validação das equações propostas pelo NRC (2001). Revista Brasileira de Zootecnia, v.32, n.2, p.480-490, 2003.

SCHOFIELD, P.; PITT, R.E.; PELL, A.N. Kinetics of fiber digestion from in vitro gas production. Journal of Animal Science, v.72, n.11, p.2980-2991, 1994.

SNEDCOR, G.W.; COCHRAN, W.G. Statistical methods. 8.ed Iowa: Iowa University Press, 1989. 503p.

TILLEY, J.M.A.; TERRY, R.A. A two-stage technique for the in vitro digestion of forage crops. Journal of British Grassland Society, v.18, n.2, p.104-111, 1963.

Van SOEST, P.J. Nutritional ecology of the ruminant. Ithaca: Cornell University Press, 1994. 476p.

VIEIRA, R.A.M.; PEREIRA, J.C.; MALAFAIA, P.A.M. et al. Simulação da dinâmica de nutrientes no trato gastrintestinal: aplicação e validação de um modelo matemático para bovinos a pasto. Revista Brasileira de Zootecnia, v.29, n.3, p.898-909, 2000.

WEISS, W.P.; CONRAD, H.R.; St.PIERRE, N.R. A theroticallybased model for predicting total digestible nutrient values of forage and concentrates. Animal Feed Science and Technology, v.39, n.1-2, p.95-110, 1992. 